\title{
Some existence results for a nonlinear fractional differential equation on partially ordered Banach spaces
}

\author{
Dumitru Baleanu ${ }^{1,2,3 *}$, Ravi P Agarwal ${ }^{4}$, Hakimeh Mohammadi ${ }^{5}$ and Shahram Rezapour ${ }^{5}$
}

\author{
*Correspondence: \\ dumitru@cankaya.edu.tr \\ ${ }^{1}$ Department of Chemical and \\ Materials Engineering, Faculty of \\ Engineering, King Abdulaziz \\ University, Jeddah, Saudi Arabia \\ ${ }^{2}$ Department of Mathematics, \\ Cankaya University, Ogretmenler \\ Cad. 14 06530, Balgat, Ankara, \\ Turkey \\ Full list of author information is \\ available at the end of the article
}

\begin{abstract}
By using fixed point results on cones, we study the existence and uniqueness of positive solutions for some nonlinear fractional differential equations via given boundary value problems. Examples are presented in order to illustrate the obtained results.
\end{abstract}

\section{Introduction}

The field of fractional differential equations has been subjected to an intensive development of the theory and the applications (see, for example, [1-6] and the references therein). It should be noted that most of papers and books on fractional calculus are devoted to the solvability of linear initial fractional differential equations on terms of special functions. There are some papers dealing with the existence of solutions of nonlinear initial value problems of fractional differential equations by using the techniques of nonlinear analysis such as fixed point results, the Leray-Schauder theorem, stability, etc. (see, for example, [7-19] and the references therein). In fact, fractional differential equations arise in many engineering and scientific disciplines such as physics, chemistry, biology, economics, control theory, signal and image processing, biophysics, blood flow phenomena and aerodynamics (see, for example, [20-23] and the references therein). The main advantage of using the fractional nonlinear differential equations is related to the fact that we can describe the dynamics of complex non-local systems with memory. In this line of taught, the equations involving various fractional orders are important from both theoretical and applied view points. We need the following notions.

Definition 1.1 $([1,4])$ For a continuous function $f:[0, \infty) \rightarrow \mathbb{R}$, the Caputo derivative of fractional order $\alpha$ is defined by

$$
{ }^{c} D^{\alpha} f(t)=\frac{1}{\Gamma(n-\alpha)} \int_{0}^{t}(t-s)^{n-\alpha-1} f^{(n)}(s) d s,
$$

where $n-1<\alpha<n, n=[\alpha]+1$ and $[\alpha]$ denotes the integer part of $\alpha$.

( 2013 Baleanu et al.; licensee Springer. This is an Open Access article distributed under the terms of the Creative Commons Attribution License (http://creativecommons.org/licenses/by/2.0), which permits unrestricted use, distribution, and reproduction in any medium, provided the original work is properly cited. 
Definition $1.2([1,4])$ The Riemann-Liouville fractional derivative of order $\alpha$ for a continuous function $f$ is defined by

$$
D^{\alpha} f(t)=\frac{1}{\Gamma(n-\alpha)}\left(\frac{d}{d t}\right)^{n} \int_{0}^{t} \frac{f(s)}{(t-s)^{\alpha-n-1}} d s \quad(n=[\alpha]+1),
$$

where the right-hand side is pointwise defined on $(0, \infty)$.

Definition $1.3([1,4])$ Let $[a, b]$ be an interval in $\mathbb{R}$ and $\alpha>0$. The Riemann-Liouville fractional order integral of a function $f \in L^{1}([a, b], \mathbb{R})$ is defined by

$$
I_{a}^{\alpha} f(t)=\frac{1}{\gamma(\alpha)} \int_{a}^{t} \frac{f(s)}{(t-s)^{1-\alpha}} d s
$$

whenever the integral exists.

Suppose that $E$ is a Banach space which is partially ordered by a cone $P \subseteq E$, that is, $x \leq y$ if and only if $y-x \in P$. We denote the zero element of $E$ by $\theta$. A cone $P$ is called normal if there exists a constant $N>0$ such that $\theta \leq x \leq y$ implies $\|x\| \leq N\|y\|$ (see [24]). Also, we define the order interval $\left[x_{1}, x_{2}\right]=\left\{x \in E \mid x_{1} \leq x \leq x_{2}\right\}$ for all $x_{1}, x_{2} \in E$ [24]. We say that an operator $A: E \rightarrow E$ is increasing whenever $x \leq y$ implies $A x \leq A y$. Also, $x \sim y$ means that there exist $\lambda>0$ and $\mu>0$ such that $\lambda x \leq y \leq \mu x$ (see [24]). Finally, put $P_{h}=\{x \in E \mid x \sim h\}$ for all $h>\theta$. It is easy to see that $P_{h} \subseteq P$ is convex and $\lambda P_{h}=P_{h}$ for all $\lambda>0$. We recall the following in our results. Let $E$ be a real Banach space and let $P$ be a cone in $E$. Let $(a, b)$ be an interval and let $\tau$ and $\varphi$ be two positive-valued functions such that $\varphi(t) \geq \tau(t)$ for all $t \in(a, b)$ and $\tau:(a, b) \rightarrow(0,1)$ is a surjection. We say that an operator $A: P \rightarrow P$ is $\tau$ - $\varphi$-concave whenever $A(\tau(t) x) \geq \varphi(t) A x$ for all $t \in(a, b)$ and $x \in P$ [13]. We say that $A$ is $\varphi$-concave whenever $\tau(t)=t$ for all $t$ [13]. We recall the following result.

Theorem 1.1 ([13]) Let E be a Banach space, let $P$ be a normal cone in $E$, and let $A: P \rightarrow P$ be an increasing and $\tau$ - $\varphi$-concave operator. Suppose that there exists $\theta \neq h \in P$ such that $A h \in P_{h}$. Then there are $u_{0}, v_{0} \in P_{h}$ and $r \in(0,1)$ such that $r v_{0} \leq u_{0} \leq v_{0}$ and $u_{0} \leq A u_{0} \leq$ $A v_{0} \leq v_{0}$, the operator $A$ has a unique fixed point $x^{*} \in\left[u_{0}, v_{0}\right]$, and for $x_{0} \in P_{h}$ and the sequence $\left\{x_{n}\right\}$ with $x_{n}=A x_{n-1}$, we have $\left\|x_{n}-x^{*}\right\| \rightarrow 0$.

\section{Main results}

We study the existence and uniqueness of a solution for the fractional differential equation

$$
D^{\alpha} u(t)+f(t, u(t))=0
$$

on partially ordered Banach spaces with two types of boundary conditions and two types of fractional derivatives, Riemann-Liouville and Caputo.

\subsection{Existence results for the fractional differential equation with the Riemann-Liouville fractional derivative}

First, we study the existence and uniqueness of a positive solution for the fractional differential equation

$$
D^{\alpha} u(t)+f(t, u(t))=0 \quad(0<\varepsilon<T, T \geq 1, t \in[\varepsilon, T], 0<\alpha<1),
$$




$$
u(\eta)=u(T) \quad(\eta \in(\varepsilon, t))
$$

where $D^{\alpha}$ is the Riemann-Liouville fractional derivative of order $\alpha$. Let $E=C[\varepsilon, T]$. Consider the Banach space of continuous functions on $[\varepsilon, T]$ with the sup norm and set $P=\left\{y \in C[\varepsilon, T]: \min _{t \in[\varepsilon, T]} y(t) \geq 0\right\}$. Then $P$ is a normal cone.

Lemma 2.1 Let $0<\varepsilon<T, T \geq 1, t \in[\varepsilon, T], \eta \in(\varepsilon, t)$ and $0<\alpha<1$. Then the problem $D^{\alpha} u(t)+f(t, u(t))=0$ with the boundary value condition $u(\eta)=u(T)$ has a solution $u_{0}$ if and only if $u_{0}$ is a solution of the fractional integral equation

$$
u(t)=\int_{\varepsilon}^{T} G(t, s) f(s, u(s)) d s
$$

where

$$
G(t, s)= \begin{cases}\frac{t^{\alpha-1}(\eta-s)^{\alpha-1}-t^{\alpha-1}(T-s)^{\alpha-1}}{\left(\eta^{\alpha-1}-T^{\alpha-1}\right) \Gamma(\alpha)}-\frac{(t-s)^{\alpha-1}}{\Gamma(\alpha)}, & \varepsilon \leq s \leq \eta \leq t \leq T, \\ \frac{-t^{\alpha-1}(T-s)^{\alpha-1}}{\left(\eta^{\alpha-1}-T^{\alpha-1}\right) \Gamma(\alpha)}-\frac{(t-s)^{\alpha-1}}{\Gamma(\alpha)}, & \varepsilon \leq \eta \leq s \leq t \leq T, \\ \frac{-t^{\alpha-1}(T-s)^{\alpha-1}}{\left(\eta^{\alpha-1}-T^{\alpha-1}\right) \Gamma(\alpha)}, & \varepsilon \leq \eta \leq t \leq s \leq T .\end{cases}
$$

Proof From $D^{\alpha} u(t)+f(t, u(t))=0$ and the boundary condition, it is easy to see that $u(t)-$ $c_{1} t^{\alpha-1}=-I_{\varepsilon}^{\alpha} f(t, u(t))$. By the definition of a fractional integral, we get

$$
u(t)=c_{1} t^{\alpha-1}-\int_{\varepsilon}^{t} \frac{(t-s)^{\alpha-1}}{\Gamma(\alpha)} f(s, u(s)) d s
$$

Thus, $u(\eta)=c_{1} \eta^{\alpha-1}-\int_{\varepsilon}^{\eta} \frac{(\eta-s)^{\alpha-1}}{\Gamma(\alpha)} f(s, u(s)) d s$ and

$$
u(T)=c_{1} T^{\alpha-1}-\int_{\varepsilon}^{T} \frac{(T-s)^{\alpha-1}}{\Gamma(\alpha)} f(s, u(s)) d s
$$

Since $u(\eta)=u(T)$, we obtain

$$
c_{1}=\frac{1}{\eta^{\alpha-1}-T^{\alpha-1}} \int_{\varepsilon}^{\eta} \frac{(\eta-s)^{\alpha-1}}{\Gamma(\alpha)} f(s, u(s)) d s-\frac{1}{\eta^{\alpha-1}-T^{\alpha-1}} \int_{\varepsilon}^{T} \frac{(T-s)^{\alpha-1}}{\Gamma(\alpha)} f(s, u(s)) d s .
$$

Hence,

$$
\begin{aligned}
u(t)= & \frac{t^{\alpha-1}}{\eta^{\alpha-1}-T^{\alpha-1}} \int_{\varepsilon}^{\eta} \frac{(\eta-s)^{\alpha-1}}{\Gamma(\alpha)} f(s, u(s)) d s-\frac{t^{\alpha-1}}{\eta^{\alpha-1}-T^{\alpha-1}} \int_{\varepsilon}^{T} \frac{(T-s)^{\alpha-1}}{\Gamma(\alpha)} f(s, u(s)) d s \\
& -\int_{\varepsilon}^{t} \frac{(t-s)^{\alpha-1}}{\Gamma(\alpha)} f(s, u(s)) d s=\int_{\varepsilon}^{T} G(t, s) f(s, u(s)) d s .
\end{aligned}
$$

This completes the proof.

Now, we are ready to state and prove our first main result.

Theorem 2.2 Let $0<\varepsilon<T$ be given and let $\tau$ and $\varphi$ be two functions on $(\varepsilon, T)$ such that $\varphi(t) \geq \tau(t)$ for all $t \in(\varepsilon, T)$. Suppose that $\tau:(\varepsilon, T) \rightarrow(0,1)$ is a surjection and $f(t, u(t)) \in$ $C([\varepsilon, T] \times[0, \infty])$ is increasing in $u$ for each fixed $t, f(t, u(t)) \leq 0$ and $f(t, \tau(\lambda) u(t)) \geq$ 
$\varphi(\lambda) f(t, u(t))$ for all $t, \lambda \in(\varepsilon, T)$ and $u \in P$. Assume that there exist $M_{1}>0, M_{2}>0$ and $\theta \neq h \in P$ such that

$$
M_{1} h(t) \leq \int_{\varepsilon}^{T} G(t, s) f(s, h(s)) d s \leq M_{2} h(t)
$$

for all $t \in[\varepsilon, T]$, where $G(t, s)$ is the green function defined in Lemma 2.1. Then the problem (2.1) with the boundary value condition (2.2) has a unique positive solution $u^{*} \in P_{h}$. Moreover, for the sequence $u_{n+1}=\int_{\varepsilon}^{T} G(t, s) f\left(s, u_{n}(s)\right) d s$, we have $\left\|u_{n}-u^{*}\right\| \rightarrow 0$ for all $u_{0} \in P_{h}$.

Proof By using Lemma 2.1, the problem is equivalent to the integral equation

$$
u(t)=\int_{\varepsilon}^{T} G(t, s) f(s, u(s)) d s
$$

where

$$
G(t, s)= \begin{cases}\frac{t^{\alpha-1}(\eta-s)^{\alpha-1}-t^{\alpha-1}(T-s)^{\alpha-1}}{\left(\eta^{\alpha-1}-T^{\alpha-1}\right) \Gamma(\alpha)}-\frac{(t-s)^{\alpha-1}}{\Gamma(\alpha)}, & \varepsilon \leq s \leq \eta \leq t \leq T, \\ \frac{-t^{\alpha-1}(-s)^{\alpha-1}}{\left(\eta^{\alpha-1}-T^{\alpha-1}\right) \Gamma(\alpha)}-\frac{(t-s)^{\alpha-1}}{\Gamma(\alpha)}, & \varepsilon \leq \eta \leq s \leq t \leq T, \\ \frac{-t^{\alpha-1}(T-s)^{\alpha-1}}{\left(\eta^{\alpha-1}-T^{\alpha-1}\right) \Gamma(\alpha)^{\prime}}, & \varepsilon \leq \eta \leq t \leq s \leq T .\end{cases}
$$

Define the operator $A: P \rightarrow E$ by $A u(t)=\int_{\varepsilon}^{T} G(t, s) f(s, u(s)) d s$. Then $u$ is a solution for the problem if and only if $u=A u$. It is easy to check that the operator $A$ is increasing on $P$. On the other hand,

$$
\begin{aligned}
A(\tau(\lambda) u)(t) & =\int_{\varepsilon}^{T} G(t, s) f(s, \tau(\lambda) u(s)) d s \\
& \geq \varphi(\lambda) \int_{\varepsilon}^{T} G(t, s) f(s, u(s)) d s=\varphi(\lambda) A u(t)
\end{aligned}
$$

for all $\lambda \in[\varepsilon, T]$ and $u \in P$. Thus, the operator $A$ is $\tau-\varphi$-concave. Since

$$
M_{1} h(t) \leq A h(t)=\int_{\varepsilon}^{T} G(t, s) f(s, h(s)) d s \leq M_{2} h(t)
$$

for all $t \in[\varepsilon, T]$, we get $A h \in P_{h}$. Now, by using Theorem 1.1, the operator $A$ has a unique positive solution $u^{*} \in P_{h}$. This completes the proof.

Here, we give the following example to illustrate Theorem 2.2.

Example 2.1 Let $0<\varepsilon<1$ be given. Consider the periodic boundary value problem

$$
\begin{aligned}
& D^{\frac{1}{3}} u(t)+\left\{g(t)+[u(t)]^{\alpha}\right\}=0 \quad(t \in[\varepsilon, 1]), \\
& u(\eta)=u(1),
\end{aligned}
$$


where $\eta \in(\varepsilon, t), g$ is continuous on $[\varepsilon, 1]$ and $\min _{t \in[\varepsilon, 1]} g(t)>0$. Put

$$
G(t, s)= \begin{cases}\frac{t^{-2 / 3}(\eta-s)^{-2 / 3}-t^{-2 / 3}(1-s)^{-2 / 3}}{\left(\eta^{-2 / 3}-1^{-2 / 3}\right) \Gamma(1 / 3)}-\frac{(t-s)^{-2 / 3}}{\Gamma(1 / 3)}, & \varepsilon \leq s \leq \eta \leq t \leq 1, \\ \frac{-t^{-2 / 3}(1-s)^{-2 / 3}}{\left(\eta^{-2 / 3}-1^{-2 / 3}\right) \Gamma(1 / 3)}-\frac{(t-s)^{-2 / 3}}{\Gamma(1 / 3)}, & \varepsilon \leq \eta \leq s \leq t \leq 1, \\ \frac{-t^{-2 / 3}(1-s)^{-2 / 3}}{\left(\eta^{-2 / 3}-1^{-2 / 3}\right) \Gamma(1 / 3)}, & \varepsilon \leq \eta \leq t \leq s \leq 1 .\end{cases}
$$

Then $\int_{\varepsilon}^{1} G(t, s) d s=\frac{t^{-2 / 3}(\eta-\varepsilon)^{1 / 3}-t^{-2 / 3}(1-\varepsilon)^{1 / 3}-(t-\varepsilon)^{1 / 3}\left(\eta^{-2 / 3}-1\right)}{\Gamma(4 / 3)\left(\eta^{-2 / 3}-1\right)}$. Now, define $\tau(t)=t, \varphi(t)=t^{1 / 3}, \gamma_{1}=$ $\min _{t \in[\varepsilon, 1]} g(t), \gamma_{2}=\max _{t \in[\varepsilon, 1]} g(t)$ and also $f(t, u)=g(t)+u^{1 / 3}$ for all $t$. Then $\tau:(0,1) \rightarrow(0,1)$ is a surjection and $\varphi(t)>\tau(t)$ for all $t \in(\varepsilon, 1)$. For each $u \geq 0$, we have

$$
\begin{aligned}
f(t, \tau(\lambda) u(t)) & =f(t, \lambda u(t))=g(t)+\lambda^{1 / 3}[u(t)]^{1 / 3} \\
& \geq \lambda^{1 / 3}\left(g(t)+[u(t)]^{1 / 3}\right)=\varphi(\lambda) f(t, u(t)) .
\end{aligned}
$$

Now, put $h \equiv 1, M_{1}=\left(\gamma_{1}+1\right) \min _{t \in[\varepsilon, 1], \eta \in[\varepsilon, 1]} \frac{-t^{-2 / 3}(1-\varepsilon)^{1 / 3}-(t-\varepsilon)^{1 / 3}\left(\eta^{-2 / 3}-1\right)}{\Gamma(4 / 3)\left(\eta^{-2 / 3}-1\right)}$ and $M_{2}=\left(\gamma_{2}+\right.$ 1) $\max _{\eta \in[\varepsilon, 1]} \frac{\varepsilon^{-2 / 3} \eta^{1 / 3}}{\Gamma(4 / 3)\left(\eta^{-2 / 3}-1\right)}$. Then we get

$$
\begin{aligned}
& \int_{\varepsilon}^{1} G(t, s)\left\{g(s)+[h(s)]^{1 / 3}\right\} d s \\
& \quad \leq \int_{\varepsilon}^{1} G(t, s)\left(\gamma_{2}+1\right) d s \\
& \quad \leq\left(\gamma_{2}+1\right) \max _{t \in[\varepsilon, 1]} \int_{\varepsilon}^{1} G(t, s) d s \leq\left(\gamma_{2}+1\right)\left(\max _{\eta \in[\varepsilon, 1]} \frac{\varepsilon^{-2 / 3} \eta^{1 / 3}}{\Gamma(4 / 3)\left(\eta^{-2 / 3}-1\right)}\right)=M_{2} h
\end{aligned}
$$

and

$$
\begin{aligned}
& \int_{\varepsilon}^{1} G(t, s)\left\{g(s)+[h(s)]^{1 / 3}\right\} d s \\
& \quad \geq\left(\gamma_{1}+1\right) \min _{t \in[\varepsilon, 1]} \int_{\varepsilon}^{1} G(t, s) d s \\
& \quad \geq\left(\gamma_{1}+1\right) \min _{t \in[\varepsilon, 1], \eta \in[\varepsilon, 1]} \frac{-t^{-2 / 3}(1-\varepsilon)^{1 / 3}-(t-\varepsilon)^{1 / 3}\left(\eta^{-2 / 3}-1\right)}{\Gamma(4 / 3)\left(\eta^{-2 / 3}-1\right)}=M_{1} h .
\end{aligned}
$$

Thus, by using Theorem 2.2, the problem has a unique solution in $P_{h}=P_{1}$.

\subsection{Existence results for the fractional differential equation with the Caputo fractional derivative}

Here, we study the existence and uniqueness of a positive solution for the fractional differential equation

$$
\begin{aligned}
& { }^{c} D^{\alpha} u(t)+f(t, u(t))=0 \quad(t \in[0, T], T \geq 1,1<\alpha<2), \\
& u(0)=\beta_{1} u(\eta), \quad u(T)=\beta_{2} u(\eta) \quad\left(\eta \in(0, t), 0<\beta_{1}<\beta_{2}<1\right),
\end{aligned}
$$

where ${ }^{c} D^{\alpha}$ is the Caputo fractional derivative of order $\alpha$. Let $E=C[0, T]$ be the Banach space of continuous functions on $[0, T]$ with the sup norm and

$$
P=\left\{y \in C[0, T]: \min _{t \in[0, T]} y(t) \geq 0\right\}
$$


It is known that $P$ is a normal cone. Similar to the proof of Lemma 2.1, we can prove the following result.

Lemma 2.3 Let $1<\alpha<2, T \geq 1, t \in[0, T], \eta \in(0, t)$ and $0<\beta_{1}<\beta_{2}<1$. Then the problem ${ }^{c} D^{\alpha} u(t)+f(t, u(t))=0$ with the boundary value conditions $u(0)=\beta_{1} u(\eta)$ and $u(T)=\beta_{2} u(\eta)$ has a solution $u_{0}$ if and only if $u_{0}$ is a solution of the fractional integral equation $u(t)=$ $\int_{0}^{T} G(t, s) f(s, u(s)) d s$, where

$$
G(t, s)= \begin{cases}\frac{\left[\beta_{1} T+t\left(\beta_{2}-\beta_{1}\right)\right](\eta-s)^{\alpha-1}+t(T-s)^{\alpha-1}-T(t-s)^{\alpha-1}}{T \Gamma(\alpha)}, & 0 \leq s \leq \eta \leq t \leq T, \\ \frac{t(T-s)^{\alpha-1}-T(t-s)^{\alpha-1}}{T \Gamma(\alpha)}, & 0 \leq \eta \leq s \leq t \leq T, \\ \frac{t(T-s)^{\alpha-1}}{T \Gamma(\alpha)}, & 0 \leq \eta \leq t \leq s \leq T .\end{cases}
$$

Theorem 2.4 Let $T \geq 1$ be given and let $\tau$ and $\varphi$ be two positive-valued functions on $(0, T)$ such that $\varphi(t) \geq \tau(t)$ for all $t \in(0, T)$. Suppose that $\tau:(0, T) \rightarrow(0,1)$ is a surjection and $f(t, u(t)) \in C([\varepsilon, T] \times[0, \infty])$ is increasing in u for each fixed $t, f(t, u(t))=0$ whenever $0<$ $\eta<s<t<T$ and $f(t, u(t)) \geq 0$ otherwise, and also $f(t, \tau(\lambda) u(t)) \geq \varphi(\lambda) f(t, u(t))$ for all $t, \lambda \in$ $(0, T)$ and $u \in P$. Assume that there exist $M_{1}>0, M_{2}>0$ and $\theta \neq h \in P$ such that

$$
M_{1} h(t) \leq \int_{0}^{T} G(t, s) f(s, h(s)) d s \leq M_{2} h(t)
$$

for all $t \in[0, T]$, where $G(t, s)$ is the green function defined in Lemma 2.3. Then the problem (2.3) with the boundary value conditions (2.4) has a unique positive solution $u^{*} \in P_{h}$. Moreover, for the sequence $u_{n+1}=\int_{\varepsilon}^{T} G(t, s) f\left(s, u_{n}(s)\right) d s$, we have $\left\|u_{n}-u^{*}\right\| \rightarrow 0$ for all $u_{0} \in P_{h}$.

Proof It is sufficient to define the operator $A: P \rightarrow E$ by

$$
A u(t)=\int_{0}^{T} G(t, s) f(s, u(s)) d s
$$

Now, by using a similar proof of Theorem 2.2, one can show that $A u(t) \geq 0$ for all $u \in P$ and $t \in[0, T]$, and also the operator $A$ is $\tau$ - $\varphi$-concave. By using Theorem 1.1, the operator $A$ has a unique positive solution $u^{*} \in P_{h}$. This completes the proof by using Lemma 2.3.

Below we present an example to illustrate Theorem 2.4.

Example 2.2 Let $\alpha=\frac{3}{2}$. Consider the periodic boundary value problem

$$
\begin{aligned}
& { }^{c} D^{\alpha} u(t)+g(t)+[u(t)]^{\alpha}=0 \quad(t \in[0,1]), \\
& u(0)=\frac{1}{3} u\left(\frac{1}{2}\right) \quad u(1)=\frac{1}{2} u\left(\frac{1}{2}\right),
\end{aligned}
$$

where $g$ is a continuous function on $[0,1]$ with $\min _{t \in[0,1]} g(t)>0$. Put $\beta_{2}=\eta=1 / 2, \beta_{1}=1 / 3$ and

$$
G(t, s)= \begin{cases}\frac{\left[\frac{1}{3}+\frac{1}{6} t\right]\left(\frac{1}{2}-s\right)^{1 / 2}+t(1-s)^{1 / 2}-(t-s)^{1 / 2}}{\Gamma(3 / 2)}, & 0 \leq s \leq \eta \leq t \leq 1, \\ \frac{t(1-s)^{1 / 2}-(t-s)^{1 / 2}}{\Gamma(3 / 2)}, & 0 \leq \eta \leq s \leq t \leq 1, \\ \frac{t(1-s)^{1 / 2}}{\Gamma(3 / 2)}, & 0 \leq \eta \leq t \leq s \leq 1 .\end{cases}
$$


Then $\int_{0}^{1} G(t, s) d s=\frac{\left[\frac{1}{3}+\frac{1}{6} t\right]\left(\frac{1}{2}\right)^{3 / 2}+t-t^{3 / 2}}{\Gamma(5 / 2)}$. Now, define $\tau(t)=t, \varphi(t)=t^{\alpha}, \gamma_{1}=\min _{t \in[0,1]} g(t)$, $\gamma_{2}=\max _{t \in[0,1]} g(t)$ and $f(t, u)=g(t)+u^{\alpha}$. Then it is easy to see that $\tau:(0,1) \rightarrow(0,1)$ is a surjection map and $\varphi(t)>\tau(t)$ for $t \in(0,1)$. Also, we have

$$
\begin{aligned}
f(t, \tau(\lambda) u(t)) & =f(t, \lambda u(t))=g(t)+\lambda^{\alpha}[u(t)]^{\alpha} \\
& \geq \lambda^{\alpha}\left(g(t)+[u(t)]^{\alpha}\right)=\varphi(\lambda) f(t, u(t))
\end{aligned}
$$

for all $u \geq 0$. Now, put $h \equiv 1, M_{1}=\left(\gamma_{1}+1\right) \min _{t \in[0,1]} \frac{-\frac{1}{3} t\left(\frac{1}{2}\right)^{3 / 2}-t^{3 / 2}}{\Gamma(5 / 2)}$ and also $M_{2}=\left(\gamma_{2}+\right.$ 1) $\frac{\frac{5}{6}\left(\frac{1}{2}\right)^{3 / 2}+1}{\Gamma(5 / 2)}$. Then we have

$$
\begin{aligned}
& \int_{0}^{1} G(t, s)\left\{g(s)+[h(s)]^{3 / 2}\right\} d s \\
& \quad \leq \int_{0}^{1} G(t, s)\left(\gamma_{2}+1\right) d s \\
& \quad \leq\left(\gamma_{2}+1\right) \max _{t \in[0,1]} \int_{0}^{1} G(t, s) d s \leq\left(\gamma_{2}+1\right) \frac{\frac{5}{6}\left(\frac{1}{2}\right)^{3 / 2}+1}{\Gamma(5 / 2)}=M_{2} h
\end{aligned}
$$

and

$$
\begin{aligned}
\int_{0}^{1} G(t, s)\left\{g(s)+[h(s)]^{3 / 2}\right\} d s & \geq\left(\gamma_{1}+1\right) \min _{t \in[0,1]} \int_{0}^{1} G(t, s) d s \\
& \geq\left(\gamma_{1}+1\right) \min _{t \in[0,1]} \frac{-\frac{1}{3} t\left(\frac{1}{2}\right)^{3 / 2}-t^{3 / 2}}{\Gamma(5 / 2)}=M_{1} h .
\end{aligned}
$$

Thus, by using Theorem 2.4, the problem has a unique solution in $P_{h}=P_{1}$.

\section{Competing interests}

The authors declare that they have no competing interests.

\section{Authors' contributions}

Authors contributed equally in writing this article. Authors read and approved the final version of the manuscript.

\section{Author details}

'Department of Chemical and Materials Engineering, Faculty of Engineering, King Abdulaziz University, Jeddah, Saudi Arabia. ${ }^{2}$ Department of Mathematics, Cankaya University, Ogretmenler Cad. 14 06530, Balgat, Ankara, Turkey. ${ }^{3}$ Institute of Space Sciences, Magurele, Bucharest, Romania. ${ }^{4}$ Department of Mathematics, Texas A\&M University, University Blvd. Kingsville, 78363-8202, USA. ${ }^{5}$ Department of Mathematics, Azarbaidjan Shahid Madani University, Azarshahr, Tabriz, Iran.

\section{Acknowledgements}

This work is partially supported by the Scientific and Technical Research Council of Turkey. Research of the third and forth authors was supported by Azarbaidjan Shahid Madani University. Also, the authors express their gratitude to the referees for their helpful suggestions which improved final version of this paper.

Received: 9 August 2012 Accepted: 16 April 2013 Published: 3 May 2013

\section{References}

1. Kilbas, AA, Srivastava, HM, Trujillo, Jj: Theory and Applications of Fractional Differential Equations. North-Holland Mathematics Studies, vol. 204. Elsevier, Amsterdam (2006)

2. Miller, KS, Ross, B: An Introduction to the Fractional Calculus and Fractional Differential Equation. Wiley, New York (1993)

3. Oldham, KB, Spainer, J: The Fractional Calculus. Academic Press, New York (1974)

4. Podlubny, I: Fractional Differential Equations. Academic Press, New York (1999)

5. Samko, SG, Kilbas, AA, Marichev, Ol: Fractional Integral and Derivative: Theory and Applications. Gordon \& Breach, Switzerland (1993) 
6. Weitzner, H, Zaslavsky, GM: Some applications of fractional equations. Commun. Nonlinear Sci. Numer. Simul. 15, 939-945 (2010)

7. Ahmad, B, Nieto, JJ: Existence of solutions for nonlocal boundary value problems of higher-order nonlinear fractional differential equations. Abstr. Appl. Anal. 2009, Article ID 494720 (2009)

8. Al-Mdallal, M, Syam, Ml, Anwar, MN: A collocation-shooting method for solving fractional boundary value problems. Commun. Nonlinear Sci. Numer. Simul. 15, 3814-3822 (2010)

9. Belmekki, M, Nieto, JJ, Rodriguez-Lopez, R: Existence of periodic solution for a nonlinear fractional differential equation. Bound. Value Probl. 2009, Article ID 324561 (2009)

10. Baleanu, D, Mohammadi, H, Rezapour, S: Positive solutions of a boundary value problem for nonlinear fractional differential equations. Abstr. Appl. Anal. 2012, Article ID 837437 (2012)

11. Baleanu, D, Mohammadi, H, Rezapour, S: Some existence results on nonlinear fractional differential equations. Philos. Trans. R. Soc. A, Math. Phys. Eng. Sci. 371(1990), Article ID 20120144 (2013)

12. Baleanu, D, Mustafa, OG, Agarwal, RP: On the solution set for a class of sequential fractional differential equations. J. Phys. A, Math. Theor. 43(38), Article ID 385209 (2010)

13. Zhai, C-B, Cao, X-M: Fixed point theorems for $\tau-\varphi$-concave operators and applications. Comput. Math. Appl. 59 532-538 (2010)

14. Delbosco, D, Rodino, L: Existence and uniqueness for a nonlinear fractional differential equation. J. Math. Anal. Appl. 204, 609-625 (1996)

15. Hashim, I, Abdulaziz, O, Momani, S: Homotopy analysis method for fractional IVPs. Commun. Nonlinear Sci. Numer. Simul. 14, 674-684 (2009)

16. Jafari, H, Daftardar-Gejji, V: Positive solution of nonlinear fractional boundary value problems using Adomin decomposition method. J. Appl. Math. Comput. 180, 700-706 (2006)

17. Zhao, Y, Sun, SH, Han, Z: The existence of multiple positive solutions for boundary value problems of nonlinear fractional differential equations. Commun. Nonlinear Sci. Numer. Simul. 16, 2086-2097 (2011)

18. Zhang, S: The existence of a positive solution for nonlinear fractional differential equation. J. Math. Anal. Appl. 252, 804-812 (2000)

19. Zhang, S: Existence of positive solutions for some class of nonlinear fractional equation. J. Math. Anal. Appl. 278 , 136-148 (2003)

20. Agarwal, RP, Lakshmikantam, V, Nieto, JJ: On the concept of solution for fractional differential equations with uncertainty. Nonlinear Anal. 72, 2859-2862 (2010)

21. Baleanu, D, Diethelm, K, Scalas, E, Trujillo, Jj: Fractional Calculus: Models and Numerical Methods. Series on Complexity, Nonlinearity and Chaos. World Scientific, Singapore (2012)

22. Qiu, T, Bai, Z: Existence of positive solution for singular fractional equations. Electron. J. Differ. Equ. 146, 1-9 (2008)

23. Sabatier, J, Agarwal, OP, Machado, JAT: Advances in Fractional Calculus. Theorical Developments and Applications in Physics and Engineering. Springer, Berlin (2007)

24. Rezapour, S, Hamlbarani Haghi, R: Some notes on the paper 'Cone metric spaces and fixed point theorems of contractive mappings'. J. Math. Anal. Appl. 345, 719-724 (2008)

doi:10.1186/1687-2770-2013-112

Cite this article as: Baleanu et al.: Some existence results for a nonlinear fractional differential equation on partially ordered Banach spaces. Boundary Value Problems 2013 2013:112.

\section{Submit your manuscript to a SpringerOpen ${ }^{\circ}$ journal and benefit from:}

- Convenient online submission

- Rigorous peer review

- Immediate publication on acceptance

- Open access: articles freely available online

High visibility within the field

- Retaining the copyright to your article 